\title{
Microgravity Generation Using Tilting Board for Resolution Evaluation of MEMS Accelerometer
}

\author{
Motohiro Takayasu, ${ }^{1 *}$ Ippei Tsuji, ${ }^{1}$ Hiroyuki Ito, ${ }^{1}$ Daisuke Yamane, ${ }^{1}$ Shiro Dosho, ${ }^{1}$ \\ Toshifumi Konishi, ${ }^{1,2}$ Noboru Ishihara, ${ }^{1}$ Katsuyuki Machida, ${ }^{1}$ and Kazuya Masu ${ }^{1}$ \\ ${ }^{1}$ Tokyo Institute of Technology, Yokohama, Kanagawa 226-8503, Japan \\ ${ }^{2}$ NTT Advanced Technology Corporation, Atsugi, Kanagawa 243-0124, Japan
}

(Received December 6, 2017; accepted January 5, 2018)

Keywords: microgravity generation, tilting board, resolution evaluation, MEMS accelerometer

We describe microgravity generation and the resolution evaluation results of a sub-1-mG microelectromechanical system (MEMS) accelerometer $\left(1 \mathrm{G}=9.8 \mathrm{~m} / \mathrm{s}^{2}\right)$. To realize input acceleration below $1 \mathrm{mG}$, we show the principle of generating microgravity acceleration by adjusting the tilt angle. The change in acceleration induced by a tilting board is quantitatively analyzed, and the capacitance change of the MEMS accelerometer as a function of tilt angle is measured. The analytical results show that the tilting board has the potential to generate microgravity. The experimental results reveal that the sub-1-mG MEMS accelerometer can realize the detection of sub-1-mG-level acceleration.

\section{Introduction}

Microelectromechanical system (MEMS) accelerometers have been widely used in various applications such as shock detection of vehicles, active stabilization of mobile robots, and motion detection of head-mounted displays. ${ }^{(1-4)}$ High-resolution MEMS accelerometers could realize potential applications such as inertial navigation, earthquake prediction, and satellite control. $^{(5-7)}$ Thus, our group has developed MEMS accelerometers with sensing resolutions higher than $1 \mathrm{G}\left(1 \mathrm{G}=9.8 \mathrm{~m} / \mathrm{s}^{2}\right)$. $^{(8-10)}$

The performance of MEMS accelerometers is affected by noise, including the Brownian noise $B_{n} .^{(11-14)}$ In practical use, the sensing resolution of MEMS accelerometers is evaluated using vibration exciters. As a result, accelerometer evaluation depends on the performance of vibration exciters. ${ }^{(15-18)}$ There have been no vibration exciters generating microgravity acceleration because mechanical and electrical noises generate acceleration noise that is larger than microgravity. Thus, MEMS accelerometers have not been measured within a range of $10^{2} \mathrm{mG}$. To investigate the characteristics of MEMS accelerometers at the input acceleration of sub-1 $\mathrm{mG}$, we need a method of generating microgravity acceleration.

To realize input acceleration below $1 \mathrm{mG}$, we have investigated a method using a tilting board. By adjusting the tilt angles of the tilting board, this method would generate microgravity

*Corresponding author: e-mail: paper@1si.pi.titech.ac.jp

https://doi.org/10.18494/SAM.2018.1840 
acceleration. Although methods with rotation motors have been used in practice for the evaluation of MEMS inclinometers, ${ }^{(19-22)}$ microgravity acceleration cannot be applied to such methods; thus, we have developed a MEMS inclinometer fabricated with the multilayer-metal technology ${ }^{(23)}$ and a tilting-board method to evaluate the MEMS inclinometer. ${ }^{(24)}$

In this study, we investigated the possibility of generating microgravity acceleration and evaluating the sensing resolution of the developed sub-1-mG MEMS accelerometer ${ }^{(8-10)}$ by the tilting-board method. First, we describe the approach for generating microgravity acceleration using a tilting board. Next, the inertial force applied to the MEMS accelerometer set on the tilting board is analytically obtained. Finally, we present the static characteristics of the sub-1mG MEMS accelerometer determined by the tilting-board method.

\section{Principle}

\subsection{Microgravity generation}

We show the principle of generating microgravity acceleration by adjusting the tilt angle. When the MEMS accelerometer is tilted by $\theta_{x}$, the gravitational acceleration $g(g=1 \mathrm{G})$ is divided into acceleration along the sensing axis $A_{x}$ and acceleration along the y-axis $A_{y}$, as shown in Fig. 1. $A_{x}$ is defined as

$$
A_{x}=\operatorname{gcos} \theta_{x}[\mathrm{G}]
$$

The acceleration change $A_{0 x}$ is described by

$$
A_{0 x}=\mathrm{g}-\mathrm{g} \cos \theta_{x}[\mathrm{G}] .
$$

Figure 2 shows the analytical result of $A_{0 x}$ as a function of $\theta_{x}$. In Fig. 2(a), $A_{0 x}$ changes from 0 to $1 \mathrm{G}$, when $\theta_{x}$ changes from 0 to $90^{\circ}$. If $\theta_{x}$ is $0.10^{\circ}, A_{0 x}$ is $1.5 \mu \mathrm{G}$, as shown in Fig. 2(b). These results suggest that microgravity acceleration can be generated by adjusting $\theta_{x}$.

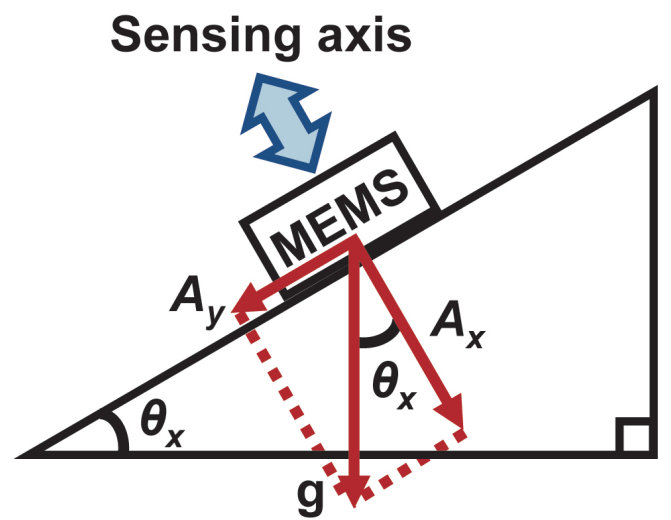

Fig. 1. (Color online) Analytical modeling of inertial force acting on a MEMS accelerometer set on a tilting board. 


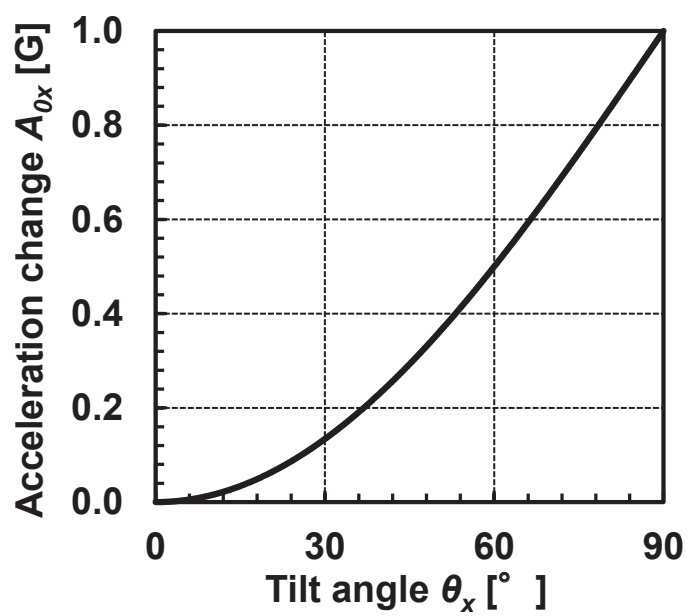

(a)

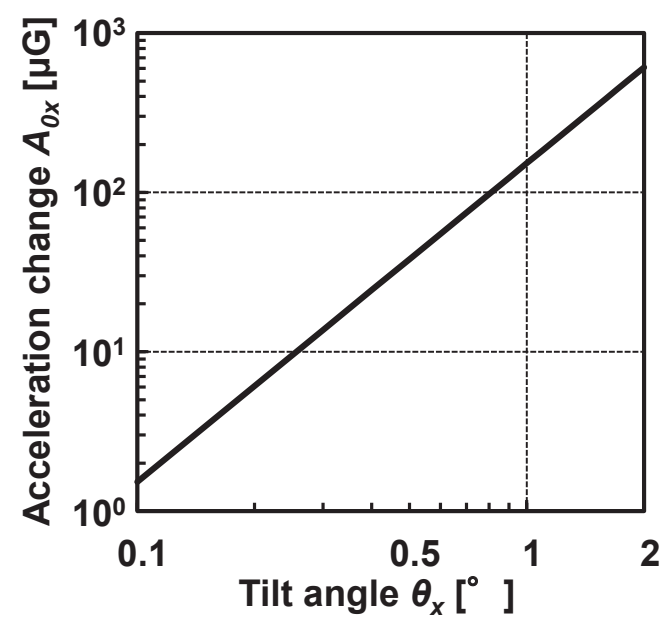

(b)

Fig. 2. Analysis results of acceleration change as a function of tilt angle in the ranges (a) from 0 to $180^{\circ}$ and (b) from 0.1 to $2^{\circ}$.

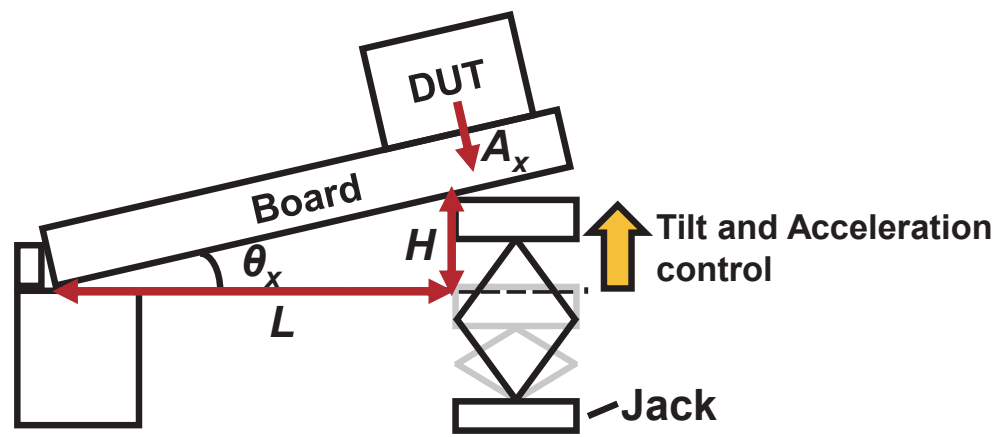

Fig. 3. (Color online) Tilting-board method.

To generate microgravity acceleration by the above-mentioned principle, an evaluation apparatus is constructed. Figure 3 shows the tilting-board method for sub-1-mG MEMS accelerometers. The MEMS accelerometer is set on the tilting board. The tilting board is composed of a jack and a board. The tilt angle $\theta_{x}$ of the tilting board can be controlled by changing the height $H$ of the jack. $\theta_{x}$ can be calculated from the distance $L$ and $H$.

$$
\theta_{x}=\tan ^{-1}\left(\frac{H}{L}\right)\left[^{\circ}\right]
$$

When Eq. (3) is substituted into Eq. (2), $A_{0 x}$ is described by

$$
A_{0 x}=\mathrm{g}-\mathrm{g} \cos \left\{\tan ^{-1}\left(\frac{H}{L}\right)\right\}[\mathrm{G}] .
$$




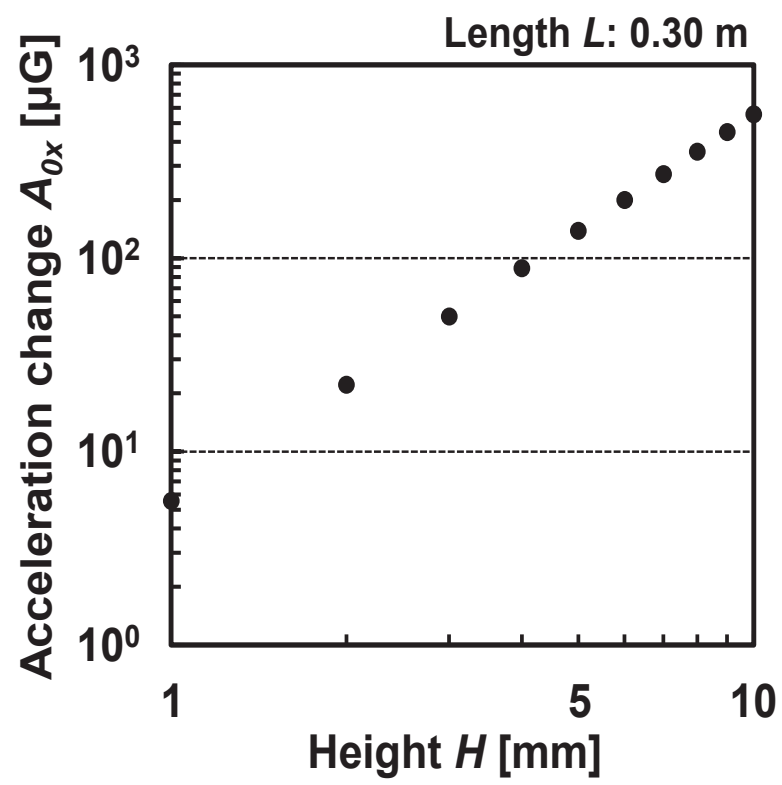

Fig. 4. Analysis results of acceleration change as a function of tilting-board height.

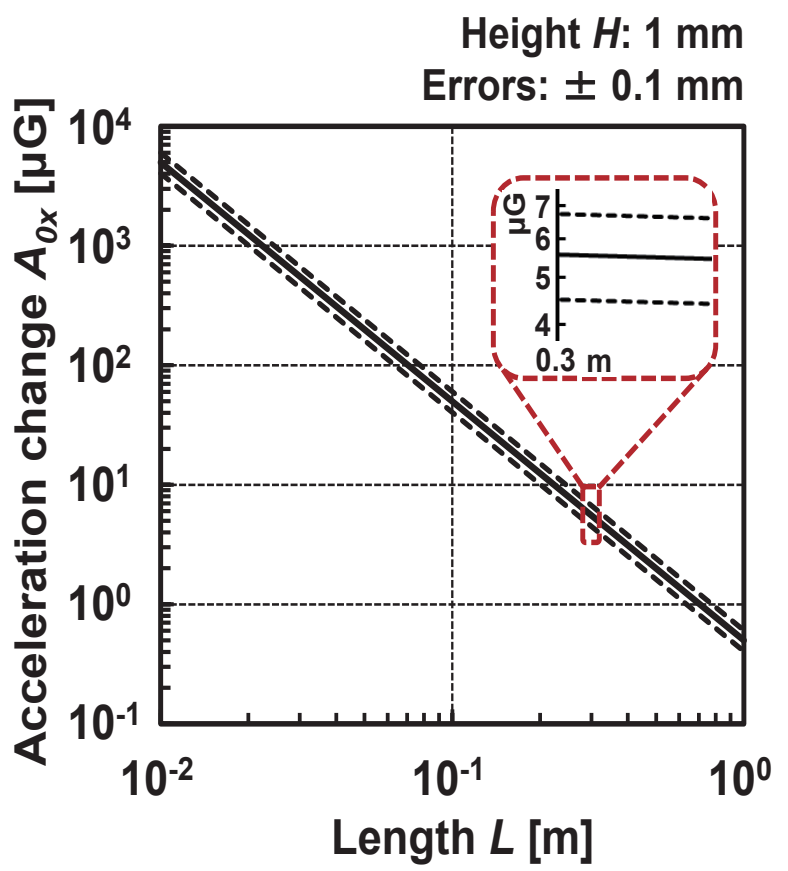

Fig. 5. (Color online) Analysis results of acceleration change as a function of tilting-board length.

In Fig. 4, $L$ is set to be $0.30 \mathrm{~m}$ and $H$ is changed in $1 \mathrm{~mm}$ steps; thereby, we can realize $A_{0 x}$ with a sub-1-mG resolution. The longer the $L$ we set, the smaller $A_{0 x}$ becomes, as shown in Fig. 5. When $L$ is set to be $1 \mathrm{~m}$ and $H$ is changed from 0 to $1 \mathrm{~mm}$ with an error of $\pm 0.1 \mathrm{~mm}, A_{0 x}$ becomes $0.5 \pm 0.1 \mu \mathrm{G}$. By this method, we could apply microgravity acceleration to the MEMS accelerometer and analyzed the capacitance change of the sub-1-mG MEMS accelerometer as a function of microgravity acceleration. 


\subsection{Detection of acceleration}

A schematic model of the sub-1-mG MEMS capacitive accelerometer is shown in Fig. $6 .^{(8-10)}$ When acceleration is applied to the MEMS accelerometer, $C_{s}$ is changed. $C_{p}$ is capacitance in the $\mathrm{SiO}_{2}$ film. The total capacitance $C_{x}$ of the MEMS accelerometer on the tilting board shown in Fig. 1 is defined by

$$
C_{x}=\varepsilon_{0} \frac{S}{d^{\prime}-\frac{9.8 m g \cos \theta_{x}}{k}}[\mathrm{~F}]
$$

where $\varepsilon_{0}, S$, and $k$ are the dielectric constant of vacuum, the proof-mass area, and the spring constant, respectively. $d^{\prime}$ is described by

$$
d^{\prime}=d_{0}+\frac{d_{1}}{\varepsilon_{2}}[\mathrm{~m}]
$$

where $d_{0}, d_{1}$, and $\varepsilon_{2}$ are the initial gap between the proof mass and the $\mathrm{SiO}_{2}$ film, the thickness of the $\mathrm{SiO}_{2}$ film, and the dielectric constant of $\mathrm{SiO}_{2}$, respectively. When $\theta_{x}$ is $0^{\circ}$, the capacitance $C_{0}$ of the MEMS accelerometer is determined by

$$
C_{0}=\varepsilon_{0} \frac{S}{d^{\prime}-\frac{9.8 m \mathrm{~g}}{k}}[\mathrm{~F}]
$$

Thus, the capacitance change $C_{0 x}$ of the MEMS accelerometer is analytically obtained by

$$
C_{0 x}=C_{0}-C_{x}=\varepsilon_{0} \frac{S}{d^{\prime}-\frac{9.8 m \mathrm{~g}}{k}}-\varepsilon_{0} \frac{S}{d^{\prime}-\frac{9.8 m \mathrm{~g} \cos \theta_{x}}{k}}[\mathrm{~F}] .
$$

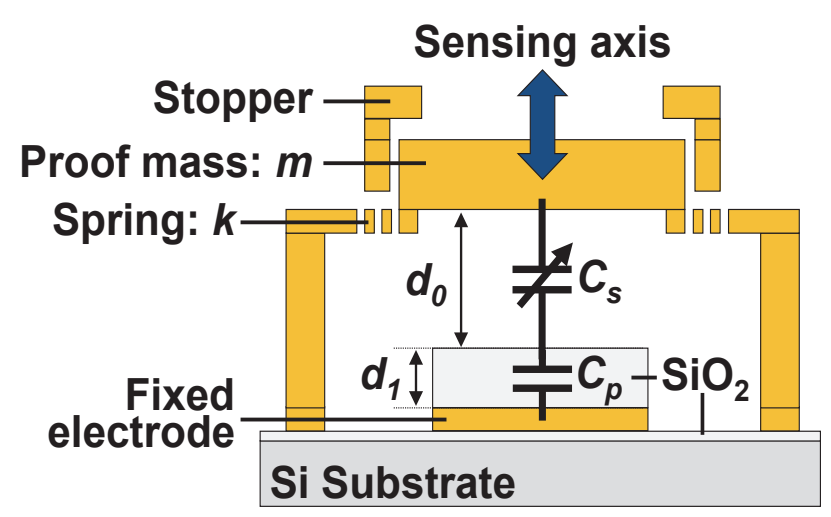

Fig. 6. (Color online) Schematic model of the sub-1-mG MEMS capacitive accelerometer. ${ }^{(8-10)}$ 


\section{Experimental Results}

To experimentally confirm the above principles, the MEMS accelerometer was fabricated by gold electroplating, as shown in Fig. 7(a). ${ }^{(8-10)}$ The proof-mass area was designed to be $2.0 \times 2.0 \mathrm{~mm}^{2}$. Each corner of the proof-mass was suspended by micromechanical suspension. A sensor module was composed of the MEMS accelerometer and a capacitance-to-digital converter (CDC; AD7745, Analog Devices), as shown in Fig. 7(b). The capacitance change of the MEMS accelerometer was obtained using the CDC. The microcontroller (Arduino Due, Arduino) received digital signals from the $\mathrm{CDC}$ and sent them to a personal computer (PC) to store the data.

To evaluate the sensing resolution of the MEMS accelerometer, the frequency response of the MEMS accelerometer was measured using the LCR meter (HIOKI E.E. IM3533-01). Figure 8 shows the capacitance and phase changes as a function of the sensing-signal frequency. The mechanical resonant frequency $f_{\text {res }}$ and quality factor $Q$ were measured to be $445 \mathrm{~Hz}$ and 3.29 , respectively. By using the results, ${ }^{(9)}$ the actual $B_{n}$ was estimated to be $398 \mathrm{nG} / \sqrt{\mathrm{Hz}}$, as summarized in Table 1. These results confirm that the sub-1-mG MEMS accelerometer has the capability to detect acceleration below $1 \mathrm{mG}$.

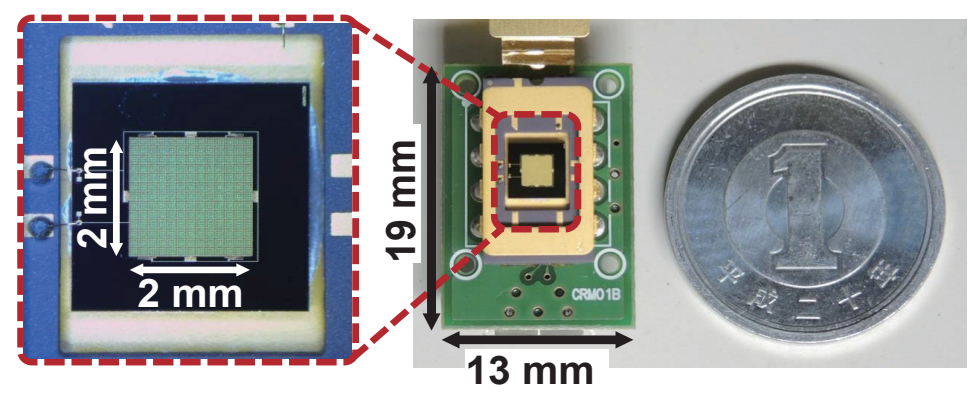

(a)

(b)

Fig. 7. (Color online) Photographs of (a) MEMS accelerometer and (b) sensor module.

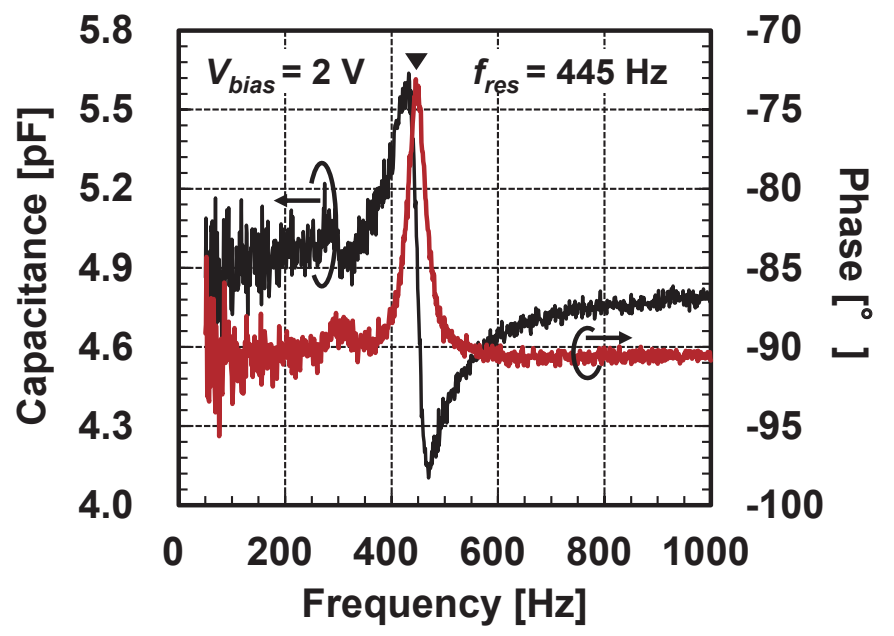

Fig. 8. (Color online) Measured capacitance and phase as a function of sensing-signal frequency. 
Table 1

Design and measured parameters of the MEMS accelerometer.

\begin{tabular}{lccc}
\hline Parameters & Design & Measured & Unit \\
\hline$m$ & $8.43 \times 10^{-7}$ & $9.27 \times 10^{-7}$ & $\mathrm{~kg}$ \\
$f_{\text {res }}$ & 262 & 445 & $\mathrm{~Hz}$ \\
$Q$ & $3.00 \times 10^{-1}$ & 3.29 & - \\
$B_{n}$ & 67.0 & 398 & $\mathrm{nG} /{ }^{\mathrm{Hz}}$ \\
\hline
\end{tabular}

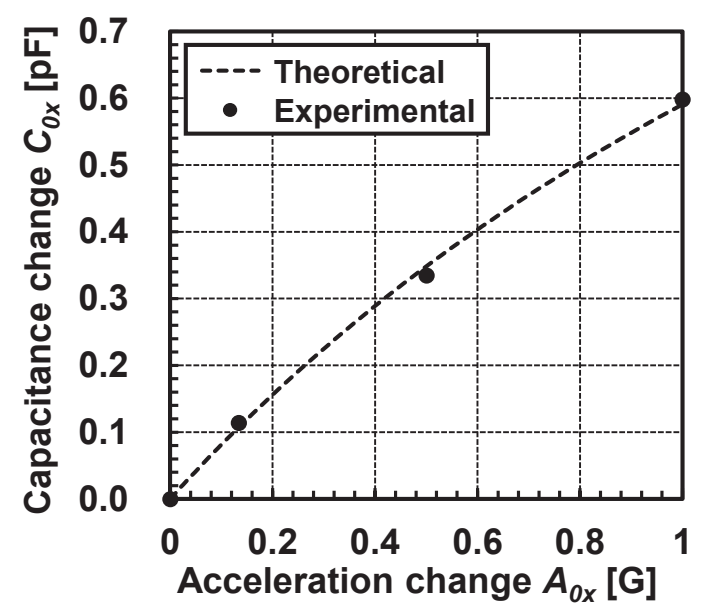

(a)

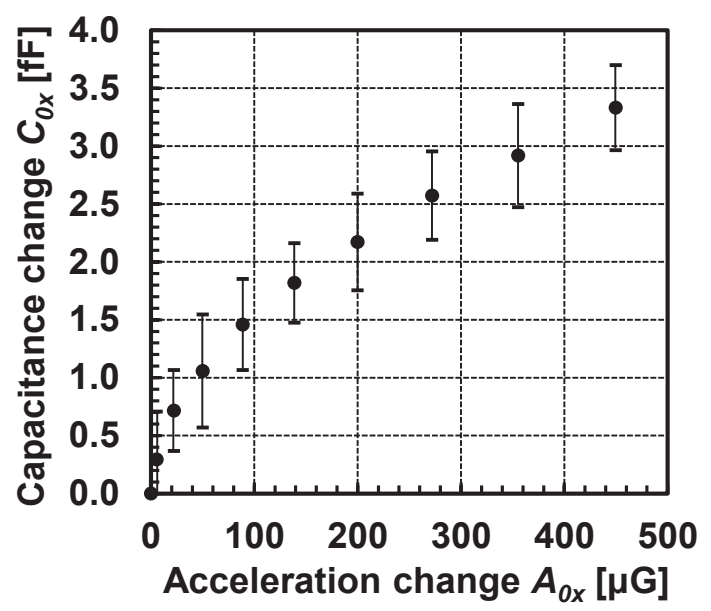

(b)

Fig. 9. Measured capacitance change as a function of acceleration change ranging (a) from 0 to $1 \mathrm{G}$ and (b) from 0 to $500 \mu \mathrm{G}$.

The microgravity characteristics of the MEMS accelerometer were evaluated by the tiltingboard method. The sensor module was set on a tilting board, where $L$ was set to be $0.30 \mathrm{~m}$. $A_{0 x}$ was calculated using Eq. (4) and measured using the reference tilt sensor (BBMO-66, MonotaRO). Figure 9(a) shows $C_{0 x}$ as a function of $A_{0 x}$ ranging from 0 to $1 \mathrm{G}$. The plotted points in Fig. 9(a) are the averaged experimental results of $C_{0 x}$, and the dashed line is the fitting curve derived using Eq. (8) with parasitic capacitances. These results indicate that the MEMS accelerometer could measure microgravity acceleration; the experimental results coincide with the theoretical model. Figure 9(b) shows the measured $C_{0 x}$ as a function of $A_{0 x}$ ranging from 0 to $500 \mu \mathrm{G}$. The plotted points in Fig. 9(b) are the average values, and the error bars show the maximum and minimum values. The results indicate that the sensor module has a potential to detect microgravity acceleration below $100 \mu \mathrm{G}$. By applying actual microgravity acceleration by the tilting-board method, we confirmed that the sub-1-mG MEMS accelerometer had the capability to detect microgravity acceleration with a sensing resolution higher than $100 \mu \mathrm{G}$.

\section{Conclusions}

We discussed the microgravity generation and resolution evaluation results of the sub-1mG MEMS accelerometer. To realize input acceleration below $1 \mathrm{mG}$, we showed the principle of generating microgravity acceleration by adjusting the tilt angle. The acceleration changes 
obtained with the tilting board were quantitatively analyzed, and capacitance changes of the MEMS accelerometer as a function of tilt angle were measured. The analytical results showed that the tilting board had the capability to generate microgravity. The experimental results revealed that the sub-1-mG MEMS accelerometer could realize the detection of sub-1-mG-level acceleration.

\section{Acknowledgments}

This work was supported by JST CREST Grant Number JPMJCR1433, Japan.

\section{References}

1 B. E. Boser and R. T. Howe: IEEE J. Solid-State Circuits 31 (1996) 366.

2 N. Yazdi, F. Ayazi, and K. Najafi: Proc. IEEE 86 (1998) 1640.

3 N. Barbour and G. Schmidt: IEEE Sens. J. 1 (2001) 332.

4 C. Acar and A. M. Shkel: J. Micromech. Microeng. 13 (2003) 634.

5 B. V. Amini and F. Ayazi: J. Micromech. Microeng. 15 (2005) 2113.

6 G. Krishnan, C. U. Kshirsagar, G. K. Ananthasuresh, and N. Bhat: J. Indian Inst. Sci. 87 (2007) 333.

7 J. Wu, G. K. Fedder, and L. R. Carley: IEEE J. Solid-State Circuits 39 (2004) 722.

8 K. Machida, T. Konishi, D. Yamane, H. Toshiyoshi, and K. Masu: ECS Trans. 61 (2014) 6.

9 D. Yamane, T. Konishi, T. Matsushima, K. Machida, H. Toshiyoshi, and K. Masu: Appl. Phys. Lett. 104 (2014) 074102.

10 D. Yamane, T. Konishi, H. Toshiyoshi, K. Masu, and K. Machida: ECS Trans. 72 (2016) 7.

11 T. B. Gabrielson: IEEE T. Electron Dev. 40 (1993) 903.

12 J. Wu, G. K. Fedder, and L. R. Carley: IEEE J. Solid-State Circuits 39 (2004) 722.

13 H. Sun, D. Fang, K. Jia, F. Maarouf, H. Qu, and H. Xie: IEEE Sens. J. 11 (2011) 925.

14 M. H. Tsai, Y. C. Liu, and W. Fang: J. Microelectromech. Syst. 21 (2012) 1329.

15 M. Lemkin and B. E. Boser: IEEE J. Solid-State Circuits 34 (1999) 456.

16 H. Kulah, J. Chae, N. Yazdi, and K. Najafi: IEEE J. Solid-State Circuits 41 (2006) 352.

17 T. Konishi, D. Yamane, T. Matsushima, G. Motohashi, K. Kagaya, H. Ito, N. Ishihara, H. Toshiyoshi, K. Machida, and K. Masu: Jpn. J. Appl. Phys. 52 (2013) 06GL04.

18 H. Xu, X. Liu, and L. Yin: IEEE J. Solid-State Circuits 50 (2015) 2101.

19 S. Luczak and W. Oleksiuk: Eng. Mech. 14 (2007) 143.

20 S. S. Yun, D. H. Jeong, S. M. Wang, C. H. Je, M. L. Lee, G. Hwang, C. A. Choi, and J. H. Lee: J. Micromech. Microeng. 19 (2009) 035025.

21 S. Luczak: Eng. Mech. 18 (2011) 341.

22 F. S. Alves, R. A. Dias, J. M. Cabral, J. Gaspar, and L. A. Rocha: J. Microelectromech. Syst. 24 (2015) 931.

23 K. Machida, S. Shigematsu, H. Morimura, Y. Tanabe, N. Sato, N. Shimoyama, T. Kumazaki, K. Kudou, M. Yano, and H. Kyuragi: IEEE Trans. Electron Devices. 48 (2001) 2273.

24 I. Tsuji, M. Takayasu, H. Ito, D. Yamane, S. Dosho, T. Konishi, N. Ishihara, K. Machida, and K. Masu: Proc. 2017 Int. Conf. Solid-State Devices and Materials (SSDM, 2017) 369. 